\title{
Moraxella catarrhalis sepsis
}

\author{
Paola Gualdi', Lucia Collini', Michele Schinella', Gabriele Mucci² \\ 'Laboratorio Patologia Clinica \\ ¿U.O. Pediatria, Ospedale S. Maria del Carmine - Rovereto (TN)
}

\section{Moraxella catarrhalis sepsis}

Key words: Sepsi, Moraxella catarrhalis

\section{SUMMARY}

Moraxella catarrhalis is a Gram-negative aerobic diplococcus, commensal of the oro-pharingeal cavity, actually playing an emerging role in the upper respiratory tract infections together with Streptococcus pneumoniae and Haemophilus influenzae. This study reports the case of a twoyearold child who was admitted into hospital with fever, headache and vomit. The chest radiograph showed signs of basal lung involvementmaking the clinical suspicion of bacterial infectionlikely.

From blood culture a Moraxella catarrhalis strain was isolated. In young children M. catarrhalis is known to cause otitis media and sinusitis, but only rarely bacteremia. In this case the child showed a clinical picture of lung infection and consequent sepsis due to M. catarrhalis.

Received February 27, 2007

Accepted April 16, 2007

\section{INTRODUZIONE}

Moraxella catarrhalis è un cocco Gram-negativo, commensale del cavo orale che a lungo è stato ritenuto dotato di scarso potere patogeno e che ora si sta affermando quale patogeno emergente nelle infezioni dell'albero respiratorio accanto a Streptococcus pneumoniae e Haemophilus influenzae, in particolare nei soggetti con broncopatia cronica ostruttiva (BPCO).

Nei soggetti immunocompromessi le infezioni che $M$. catarrhalis può causare sono molteplici (polmoniti, endocarditi, setticemia e meningiti). Attualmente $M$. catarrhalis può essere considerato anche un patogeno nosocomiale in virtù di epidemie ospedaliere descritte.

La sua valutazione come commensale del tratto respiratorio fino agli anni ' 80 si basava sul fatto che le caratteristiche microbiologiche di M. catarrhalis sono molto simili a quelle di Neisseria cinerea, nota specie appartenente alla flora microbica residente dell'apparato respiratorio. Inoltre, essendo piuttosto frequente la sua colonizzazione a livello di vie respiratorie superiori, stabilire il suo ruolo patogeno diventa difficile.

Raramente causa infezioni invasive ed è inusuale isolare M. catarrhalis da sangue o da liquidi pleurici. Nei bambini $M$. catarrhalis si colloca al terzo posto, dopo S. pneumoniae e $H$. influenzae, come agente eziologico di sinusiti e otite media.
Già dopo il quarto anno di età si possono ritrovare anticorpi sierici che si sviluppano verso proteine di membrana, tuttavia molti aspetti della reazione immunitaria dell'ospite sono ancora poco noti. Nel meccanismo di difesa entrano in gioco fattori locali a livello di vie respiratorie, ma lo sviluppo di una vera e propria risposta infiammatoria accompagnata da un movimento anticorpale specifico aumenta le difese dell'ospite.

\section{DESCRIZIONE DEL CASO CLINICO}

Si riferisce il caso di un bambino di due anni giunto in Pronto Soccorso e successivamente trasferito presso 1'Unità Operativa di Pediatria del nostro Ospedale con iperpiressia dal giorno precedente, cefalea e vomito.

I genitori riferiscono di un episodio febbrile dieci giorni prima risolto, ma poi ricomparso associato a vomito e malessere generale.

Sulla base dei sintomi e dell'esame obiettivo viene eseguita una radiografia del torace che mostra un focolaio basale. I prelievi ematici per emocromo ed esami biochimici mostrano valori alterati. All'ingresso: WB $15.3 \times 10^{9} \mathrm{~L}$, PCR $118.5 \mathrm{mg} / \mathrm{L}$, VES $98 \mathrm{~mm} / \mathrm{h}$. In seguito alla negatività della ricerca diretta degli antigeni virali, scaturisce il sospetto clinico di infezione batterica. Viene eseguita l'intradermoreazione di Mantoux, che risulta essere negativa ed un prelievo per emocoltura. 
Successivamente viene eseguita terapia con ceftriaxone in infusione venosa, con rapida risoluzione della sintomatologia e ripristino dei parametri di laboratorio.

L'emocoltura si è positivizzata dopo circa 18 ore di incubazione a $37^{\circ} \mathrm{C}$. Al vetrino eseguito dal flacone sono stati evidenziati diplococchi Gram negativi e la ricerca di antigeni batterici è risultata negativa per Neisseria meninigitidis. Dalle sottocolture eseguite in agar sangue e agar cioccolato, incubate in microaerofilia al $5 \%$ di $\mathrm{CO}_{2}$ overnight sono state isolate colonie piccole a "goccia di rugiada", semitraslucide e con positività del test ossidasi.

La colorazione di Gram eseguita sulle colonie evidenziava diplococchi Gram negativi e le prove biochimiche, eseguite con gallerie Api-NH (bioMérieux), identificavano $M$. catarrhalis. L'antibiogramma, eseguito su Muller Hinton sangue, ha consentito di rilevare in vitro resistenza a ciprofloxacina e sensibilità ad ampicillina, eritromicina, amoxicillina-acido clavulanico, cefaclor, ceftriaxone e trimethoprim-sulfametossazolo. Il ceppo risultava essere $\beta$-lattamasi non produttore.

\section{DISCUSSIONE}

Batteriemie causate da $M$. catarrhalis sono rare ma la frequenza tende ad aumentare in bambini immunocompromessi, infatti la letteratura riporta episodi sporadici con fattori di rischio predisponenti. In genere sono presenti sintomi a carico delle vie respiratorie (otite media, sinusite e polmonite) con febbre.

Nel caso da noi presentato il soggetto, immunologicamente competente, manifestava alla $\mathrm{Rx}$ torace un quadro compatibile con infezione polmonare. Presumibilmente l'agente causale era $M$. catarrhalis, isolata successivamente in campioni di sangue.

In letteratura viene riportato che la maggior parte dei ceppi isolati sono produttori di ß-lattamasi, diventa quindi importante non solo il suo riscontro nei campioni clinici ma anche il saggiare un profilo di sensibilità antibiotica al fine di impostare una corretta e mirata terapia.

In conclusione, il nostro caso sottolinea l'importanza di questo agente eziologico sul piano clinico coerentemente ad altri dati della letteratura scientifica più recente.

\section{BIBLIOGRAFIA}

1. Abuhammour WM, Abdel-Haq NM, Asmar BI, Dayani AS. Moraxella catarrhalis: bacteremia: a 10year experience. South Med J 1999; 92(11): 1071-4.

2. Ernst-Kruise MR, Ruthgers MI, Revesz T, Wolfs TF, Fleer A, Geelen SP. Invasive infection with Moraxella catarrhalis in two children with lymphatic leukaemia and granulocytopenia. Ned Tijdschr Geneeskd. 2003; 147(23): 1126-8.

3. Ohkusu K, Nakamura A, Horie H, Udagawa A. Fatal sepsis with acute pancreatitis caused by Moraxella catarrhalis in a child. Pediatr Infect Dis J 2001; 20(9): 914-5.

4. Owens RC, Tessier P, Nightingtale CH, Ambrose PG, Quintiliani R, Nicolau DP. Pharmacodynamics of ceftriaxone and cefexime against community-acquired respiratory tract pathogens. International Journal of Antimicrobial Agents 2001; 17: 483-9.

5. Paykel JM. Moraxella (Branhamella) catarrhalis infections. Honorable mention manuscript 2002; 9: 33-5.

6. Quinones R, Llanes R, Torano G, Perez M. Nasopharingeal colonization by and study of antimicrobial susceptibility in healthy children from Cuban day-care centers. Archives of medical research 2005; 36: $80-2$.

7. Richards SJ, Greening AP, Enright MC, Morgan MG, Mckenzie H. Outbreak of Moraxella catarrhalis in a respiratory unit. Thorax 1993; 48: 91-6.

8. Sugiyama H, Ogata E, Shimamoto Y, et al. Bacteremic Moraxella catarrhalis in a patient with immunoglobulin deficiency. J Infect Chemother 2000; 6(1): 61-2.

9. Thornsberry C, Jones M, Hickey M, Mauriz Y, Kahn J, Sahm D. Resistance surveillance of Streptococcus pneumoniae, Haemophilus influenzae and Moraxella catarrhalis isolated in the United States 1997-1998. J Antimicrob Chemoter 1999; 45: 457-66.

10. Verduin CM, Hall C, Fleer A, Vandijk H, Vanbelkum A. Moraxella catarrhalis: from emerging to established pathogen. Clinical Microbiology Reviews 2002; 15: 125-44. 